\title{
Zur Rezeption tschechischer Kinder- und Jugendliteratur im deutschen Sprachraum \\ Preisgekrönte Werke
}

\author{
Jana Baroková
}

\section{Einführung}

Gegenstand dieses Beitrags sind Betrachtungen über den Stand der tschechischen Kinder- und Jugendliteratur in Deutschland im Zeitraum von 1945-1989. Obwohl es damals den gemeinsamen Staat der Tschechen und Slowaken gab, widme ich mich hier ausschließlich der tschechischen Kinder- und Jugendliteratur. Ich konzentriere mich auf die Gattung der Prosa, die Rezeption der tschechischen Kinderlyrik bleibt für die Zwecke des vorliegenden Beitrags ausgeschlossen.

Die tschechische Literatur wurde von den Literaturen der ehemaligen Ostblockstaaten hinter dem sog. Eisernen Vorhang oft rezipiert; sie galt als innovativ und stilbildend. Neben der Literatur waren vor allem drei andere Medien stark vertreten, die entscheidend zur großen Popularität der tschechischen Kinderliteratur beitrugen: das Theater mit dem bekannten Marionettenpaar Spejbl und Hurvínek, der Film mit Josef Ladas Kocour Mikeš und letztendlich das Fernsehen. Vor allem das Fernsehen spielte mit verschiedenen TV-Serien und Kino- bzw. Fernsehfilmen eine wichtige Rolle. An den tschechischen Filmen wurden vor allem der innovative Ansatz der Drehbuchautoren und Regisseure geschätzt sowie der Umstand, dass diese sich häufig durch das tradierte Volksdichtungsgut der alten Märchen inspirieren ließen. Ihre Vorliebe zur Kombination diverser Stile und die große Phantasie ihrer Werke waren hoch geschätzt. Seit den sechziger Jahren fand der tschechische Film - verbunden vor allem mit Namen wie Hermína Týrlová, Jiř́ Trnka oder Karel Zeman - weltweit Anerkennung. Die Werke von Ota Hofman und Jindřich Polák, wie Pan Tau, Lucie postrach ulice (Luzie, der Schrecken der Straße), Chobotnice z 2. patra (Die Tintenfische aus dem zweiten Stock) oder Návštévnici (Die Besucher), wurden zu Kultfilmen. Kinder in den beiden damaligen deutschen Staaten kannten den Fernsehfilm Tři oř̌̌šky pro Popelku (Drei Haselnüsse für Aschenbrödel) und er war auch bei Erwachsenen beliebt. Im Unterschied zu den anderen Ostblockliteraturen wurde die tschechische Kinder- und Jugendliteratur als westlich orientiert angesehen und hatte auch die meisten preisgekrönten Bücher. Von den Illustratoren wurden vor allem Josef Lada, Josef Čapek, Adolf Zábranský, Ota Janeček, Zdeněk Miler, Adolf Born oder Květa Pacovská hoch bewertet. (vgl. SEIFERT/WEINKAUFF: 2006).

Verglichen mit anderen Nationalliteraturen publizierten viele renommierte tschechische Schriftsteller auch vor 1968 erfolgreich sowohl für Erwachsene als 
auch für Kinder. Zu diesen gehörten Schriftsteller und Dichter wie die Brüder Karel und Josef Čapek, Jan Drda, František Halas, Bohumil Hrabal, Marie Majerová, Jiří Wolker, Oldřich Mikulášek u. a.

\section{2. Übersetzungsgeschichte}

Die tschechisch-deutschen Beziehungen sind durch die geographische Nähe und eine teilweise gemeinsame Geschichte von Zusammenarbeit aber auch von Konflikten geprägt. Seit 1945 sind vor allem zwei Jahreszahlen entscheidend: Neben dem Jahr nach der Niederschlagung des Prager Frühlings (1968) ist auch das Jahr nach der Samtenen Revolution (1989) ausschlaggebend, als das tschechische Literatursystem in mehrere Teile zerfiel. Das Werk der Autoren, die zur Emigration gezwungen waren, wie Jiř́ Gruša, Pavel Kohout, Jiří Kolár, Jan Procházka, Iva Procházková, Vladimír Škutina, Jan Vladislav, Scheila Och u. a., wurde erst gar nicht aus dem Tschechischen ins Deutsche übersetzt, denn sie schufen es infolge ihrer neuen Lebensumstände in anderen Sprachen als ihrer Muttersprache. Die in Berlin lebende Scheila Och (1940-1999) z. B. sprach bei ihrer Emigration kaum Deutsch, später (1998) veröffentlichte sie auf Deutsch ein Kinder- und Jugendbuch (Karel, Jarda und das wahre Leben), dem ein Jahr später, kurz vor ihrem Tod, das Buch Balaban, Neumann, der Hund folgte. Jan Procházka, der unter dem sozialistischen Regime Schreibverbot hatte, veröffentlichte in der Emigration deutsche Übersetzungen seiner Werke. Auch die Bücher seiner Tochter Iva Procházková oder diejenigen von Ivan Klíma wurden in der Tschechoslowakei erst nach 1989 retrospektiv publiziert. Einen weiteren Faktor stellte im Zeitraum von 1949 bis 1990 die Existenz zweier deutscher Staaten dar. Die nach 1968 in der ČSSR verbotenen Autoren wurden in der DDR nicht übersetzt, dagegen wurden sie in der damaligen BRD gerade aus diesem Grund begünstigt. Auch wegen der Aufnahme vieler tschechischer Exilautoren in die internationale „Gesellschaft der Freunde des Buches" wuchs die internationale Anerkennung der tschechoslowakischen Kinder- und Jugendliteratur ständig. Dem Schriftsteller Bohumil Ř́ha wurde im Jahre 1980, dem Illustrator Jiř́ Trnka 1968 und Květa Pacovská 1992 die Hans-ChristianAndersen-Medaille verliehen. Dieser seit 1956 existierende internationale Preis bezeichnet auch als „kleiner Nobelpreis“ - wird alle zwei Jahre an einen lebenden Schriftsteller in Form einer Medaille verliehen, seit 1966 werden auch hervorragende Illustratoren preisgekrönt (vgl. MORANSEE: 1982).

\subsection{Die Rezeption der tschechischen Kinder- und Jugendliteratur von 1945 bis 1968}

In den ersten Jahren nach dem Zeiten Weltkrieg waren Nachahmungen von Volksmärchen der tschechischen Schriftsteller des 19. Jahrhunderts wie Karel Jaromír Erben oder Božena Němcová sehr beliebt. Eines der Volksmärchen von 
Božena Němcová wurde auch als Vorlage für den Märchenfilm Drei Haselnüsse für Aschenbrödel verwendet. Sowohl in Österreich als auch in der Schweiz wurde die tschechische Kinder- und Jugendliteratur der oben genannten Autoren als Lesestoff in den Schulen verwendet. Neben dem Werk von Božena Němcová wurde auch das von Karel Čapek, Marie Majerová und Ludvík Aškenazy ins Deutsche übersetzt. Darüber hinaus ist in diesem Zusammenhang ein Werk zu nennen, das noch vor 1945 ins Deutsche übersetzt wurde: das Tierbuch Broučci (dt. Leuchtkäferchen für große und kleine Kinder) des Autors Jan Karafiát, dessen erste deutsche Übersetzung bereits auf das Jahr 1928 datiert. Am bekanntesten ist diese Allegorie auf die menschliche Gesellschaft in der Ausgabe von 1969, die - illustriert von Jiří Trnka - unter dem Titel Käferchen erschien. Dasselbe Buch parodierte einige Jahre später Ondřej Sekora in seiner lustigen Tiergeschichte Ferda mravenec, von der alle Fortsetzungen ins Deutsche übersetzt wurden.

Eine wichtige Persönlichkeit in diesem Zeitabschnitt ist Josef Lada (1887-1957), Maler, Karikaturist und Illustrator, dessen Name oft im Zusammenhang mit dem Werk Kocour Mikeš genannt wird. Die deutschsprachige Version mit dem Titel Kater Mikesch, übersetzt von Otfried Preußler, wurde 1963 mit dem Deutschen Jugendbuchpreis ${ }^{1}$ ausgezeichnet. Nachdem dieses Werk verfilmt wurde, ist seine Popularität in den deutschsprachigen Ländern noch gestiegen.

Ein weiterer wichtiger Autor dieser Periode ist Vladislav Vančura (1891-1942), Arzt, Schriftsteller, Dramatiker, Publizist, Drehbuchautor und Regisseur, der wegen seiner Beteiligung am Widerstandskampf links orientierter Intellektueller 1942 von den Nationalsozialisten hingerichtet wurde. Sein literarisches Werk ragt durch einen hoch poetischen, monumentalen Stil und eine imaginative Sprache hervor. Seine phantastische Geschichte für Kinder Kubula a Kuba Kubikula (1931) erschien deutsch 1963 unter dem Titel Peterpetz und Peter Petermichel.

Als dritter Autor in dieser Reihe ist Ludvík Aškenazy (1921-1986), der Schwiegersohn von Heinrich Mann, zu nennen. Nach dem Publikationsverbot von 1968 emigrierte er, was zur Folge hatte, dass seine Bücher nach diesem Datum nur auf Deutsch verlegt wurden. Seine sog. „Kinderetüden“ (tsch. Dětské etudy) Gespräche zwischen dem Vater und seinem Sohn - erschienen bereits Ende der fünfziger Jahre zweimal auf Deutsch.

In den fünfziger und sechziger Jahren erfreuten sich im deutschen Sprachraum zwei tschechische Kinderbücher großer Beliebtheit, und zwar Václav Řezáčs (19011956) Detektivgeschichte Haltet den Dieb (tsch. Kluci, hurá za ním, mit Illustrationen von Josef Čapek), und Josef Veromír Plevas (1899-1985) Kinderbuch-Trilogie Die Geschichte vom kleinen Bobesch (tsch. Maly Bobeš). Sowohl der erste Teil dieses Buches als auch seine Fortsetzung mit dem Titel Bobesch zieht in die Stadt erschienen in

$\overline{1}$ Dieser Preis besteht seit 1956. Ausgezeichnet werden damit deutschsprachige Originalwerke lebender Autoren ebenso wie deutsche Übersetzungen von fremdsprachigen Werken lebender Autoren, die innerhalb der letzten fünf Jahre erschienen sind. 
Zusammenarbeit eines Prager und eines Berliner Kinderbuchverlages. Nicht nur das Werk von Josef Věromír Pleva, sondern auch das von František Langer (1888-1965) ließ sich vom kinderliterarischen Schaffen Erich Kästners (1899-1974) und dessen „Großstadtgeschichten“ inspirieren. Das Buch von F. Langer Die Bruderschaft vom Weißen Schlüssel (tsch. Bratrstvo bilého kliče) erschien zuerst 1949 in Österreich, 1958 dann nochmals im deutschen Artia-Verlag.

Besondere Aufmerksamkeit erregte das Genre des Abenteuerbuches und die populärwissenschaftliche Kinderliteratur. Der Polarforscher und Schriftsteller František Běhounek (1898-1973), der in seinen Büchern den Kindern Wissenschaft populär vermitteln wollte, verfasste als Mitglied der Polarexpedition von A. Nobile einige Bücher mit dieser Thematik, die im deutschen Sprachraum später als Anthologie veröffentlicht wurden. Als ein weiterer Autor der Genres „Abenteuerbuch “ und „historische Tiergeschichte“ ist Jaromír Tomeček (1906-1997) zu nennen, dessen Werke über das Leben in der Natur Ende der sechziger Jahre deutsch verlegt wurden.

Nach 1945 erschienen in der damaligen ČSSR viele kinder- und jugendliterarische Werke, die die Thematik der Bewältigung des Zweiten Weltkrieges und des antifaschistischen Widerstandskampfes bearbeiteten. Diese Werke wurden nur in der ehemaligen DDR veröffentlicht. Das erste dieser Bücher stammt von Jan Drda (1915-1970). Es erschien 1960 unter dem Titel Schüsse an der Troja-Brücke (tsch. Jednou v máji) und befasst sich mit dem Prager Aufstand vom 5. Mai 1945. Ein weiteres wichtiges Werk, das auch auf Deutsch verfilmt wurde, ist die Erzählung Romeo und Julia und die Finsternis (1962, tsch. Romeo, Julie a tma) von Jan Otčenášek (1924-1979), die die Geschichte einer tragischen Liebe zwischen einem jüdischen Mädchen und einem jungen Tschechen erzählt, der das Mädchen während der Okkupation in der Wohnung seiner Eltern versteckt.

\subsection{Die Rezeption der tschechischen Kinder- und Jugendliteratur in den Jahren 1968 bis 1989}

Die Konsequenzen der Zerschlagung des Prager Frühlings (1968) waren auch für die Entwicklung der Kinder- und Jugendliteratur in der damaligen ČSSR dramatisch. Allmählich wurde die Kulturszene gesäubert, ein Prozess, der von offizieller Seite „Konsolidierung “ und „Normalisierung“" genannt wurde. Betroffen waren vor allem Mitglieder des tschechischen Schriftstellerverbandes. Viele Autoren gingen ins Ausland und gründeten dort Exil-Verlage. Der bekannteste dieser Verlage waren die Sixty-Eight-Publishers im kanadischen Toronto, geleitet von Josef Škvorecký (*1924) und seiner Ehefrau Zdena Salivarová $\left({ }^{*} 1933\right)$. Systemkritische Autoren, die im Land blieben, sammelten sich um den sog. „Samisdat“ (was auf Deutsch soviel wie "Selbstverlag" bedeutet). Beide Literaturszenen wurden von den offiziellen Stellen tabuisiert. Viele gute Werke, die im Ausland entstanden, blieben bis 1989 sowohl 
in der DDR als auch in der ČSSR unbekannt. Ins Exil gingen Autoren wie Ludvík Aškenazy, Jaroslav Foglar, Pavel Kohout, Jan Procházka, Iva Procházková, Scheila Och u. a. Jan Procházkas bedeutender Antikriegsroman Es lebe die Republik (tsch. At žije republika, siehe unten) wurde in der BRD bei Hans-Joachim Gelberg verlegt und 1969 mit dem Deutschen Jugendliteraturpreis ausgezeichnet.

\subsubsection{Rezeption der tschechischen Kinder- und Jugendliteratur in der DDR}

In der DDR wurden nach 1970 primär Bücher tschechischer Klassiker wie Eduard Bass, Karel Čapek, Jaroslav Hašek, Václav Čtvrtek, Božena Němcová u. a. verlegt. Das Werk der nach 1968 verbotenen Autoren durfte hier nämlich nicht veröffentlicht werden. Von den literarischen Genres erfreuten sich vor allem das Märchen und die Sage großer Popularität, weil mit der Aktualisierung der Themen des Volksdichtungsgutes an die folkloristische Tradition der Nationen der Tschechen, Mährer und Slowaken angeknüpft werden sollte. 1976 wurden im Altberliner Verlag Werke von Alena Santarová (siehe unten) und Valja Stýblová verlegt. In der Gattung „Mädchenbuch“ war sowohl im Osten als auch im Westen Stanislav Rudolf bekannt, die Gattung „Tiergeschichte“ war mit dem Werk von Jaromír Tomeček vertreten. In den siebziger und achtziger Jahren wurden Werke von Ota Hofman, wie z. B. Lucie und die Wunder (tsch. Lucie a zázraky) rezipiert, jedoch vor allem in der BRD. Letztendlich ist in diesem Zusammenhang Václav Čtvrtek (1911-1976) zu nennen. Seine Geschichte Räuber Fürchtenix (tsch. O loupežníku Rumcajsovi) erzählt von einem gutmütigen Räuber, seiner Frau Manka und dem Sohn Cipísek, die in einer Welt von märchenhaften Figuren ihre Abenteuer erleben.

\subsubsection{Rezeption der tschechischen Kinder- und Jugendliteratur in der BRD}

Verbotene Autoren bzw. diejenigen, die aus politischen Gründen emigrierten, wurden in der BRD positiv aufgenommen. Dies waren z. B. Jan Procházka, Iva Procházková, Jiří Koláŕ, Vladimír Škutina, Jan Vladislav oder Pavel Kohout. Ein Science-FictionRoman des im Schweizer Exil lebenden Autors Luděk Pešek (1919-1999), Die Erde ist nah: die Marsexpedition, wurde 1970 mit dem Deutschen Jugendliteraturpreis ausgezeichnet. Zunächst wurde das Werk der Klassiker der tschechischen Kinderliteratur bevorzugt, wie Josef Čapeks Geschichten vom Hündchen und vom Kätzchen (1982) oder Volksmärchen von Karel Jaromír Erben. Nach dem Erfolg von Jan Procházkas Roman Es lebe die Republik! wurden auch weitere Werke dieses Schriftstellers verlegt, einige von ihnen waren bereits vorher in deutscher Sprache erschienen. Unter den deutschen Verlagen, die das Werk tschechischer Schriftsteller veröffentlichten, taten sich besonders Dausien, Oetinger, Beltz \& Gelberg und der Deutsche Taschenbuchverlag hervor. Das Genre „phantastische Geschichte“ war 
besonders durch die Werk des Schriftstellers und Drehbuchautors Miloš Macourek vertreten, von denen Der fliegende Ferdinand und Arabela am bekanntesten sind.

Das Werk von Ota Hofman, das seit den 60er Jahren in der DDR beliebt war, wurde in Form der tschechischen Verfilmungen nun auch im Westen rezipiert. Am bekanntesten ist die Fernsehserie Pan Tau, die von einem geheimnisvollen Herrn in Frack handelt, der mit seinem schwarzen Hut zaubern kann. Die aus der Zusammenarbeit von Ota Hofman mit dem Regisseur Jindřich Polák entstandene Fernsehserie Luzie, der Schrecken der Straße (tsch. Lucie, postrach ulice), in der Knetfiguren die phantastische Welt darstellen sollen, wurde 1981 in Deutschland preisgekrönt. Das letzte bedeutende Werk der oben genannten Autoren ist die in der ČSSR bekannteste Science-Fiction-Serie mit dem Titel Die Besucher (tsch. Návštévníci, 1977), die in einer Koproduktion der ČSSR mit Deutschland, Frankreich und der Schweiz entstand.

Da in der zweiten Hälfte des 20. Jahrhunderts Fernsehen und Film die wichtigsten Medien der Massenkommunikation waren, kam es dazu, dass einige Kinderbücher zunächst als Fernsehserien bzw. als Zeichentrickfilme weltweit bekannt wurden. Eine der bekanntesten dieser Serien ist die Verfilmung des Kinderbuchs Wie der Maulwurf zu seinen Hosen kam, (tsch. Jak krtek ke kalhotkám priš̌el, 1957) von Zdeněk Miler, in der der Protagonist ein Maulwurf mit den Eigenschaften eines Kindes ist.

In den nächsten Kapiteln widme ich mich ausführlicher dem Leben und Schaffen dreier Autoren (Jan Procházka, Iva Procházková und Alena Santarová), deren Werk sowohl in der DDR als auch in der BRD rezipiert wurde.

\section{Jan Procházka und sein Werk}

Jan Procházka (1929-1971) war zunächst als Parteifunktionär politisch tätig und arbeitete dann als Drehbuchautor für die staatliche tschechische Filmproduktionsfirma. 1969 wurde er wegen seiner reformerischen Einstellung aus der Kommunistischen Partei der Tschechoslowakei und aus dem Schriftstellerverband ausgeschlossen. In seiner Erzählung Lenka (1969) schildert er die Probleme und den Alltag in der sozialistischen Gesellschaft. Seine Tochter Iva Procházková (geb. 1953) ist Kinderbuchautorin, ihr erstes Kinderbuch wurde 1980 im Verlag Albatros veröffentlicht. 1983 emigrierte sie mit ihrem Mann nach Österreich, später nach Deutschland. Hier widmete sie sich mit ihrem Mann dem Autorentheater, später schrieb sie Drehbücher und Theaterstücke für Kinder und Erwachsene. Zurzeit lebt und arbeitet sie in Tschechien (Kinderbuch Karolina, Albatros 1999).

Jan Procházka widmete seine autobiographischen Erinnerungen an ein südmährisches Dorf im Werk Es lebe die Republik einem der bekanntesten tschechischen Regisseure der Gegenwart: Karel Kachyňa.

Der Protagonist des Romans, Olin (Koseform von Oldřich), lebt mit seinen Eltern in einem kleinen südmährischen Dorf. Als der Zweite Weltkrieg endet 
und die Rote Armee sich dem Dorf nähert, schickt der Vater seinen Sohn in den Wald, um das Pferd vor den Soldaten zu verstecken. Olin findet einen scheinbar ruhigen und stillen Ort am Fluss. Es überraschen ihn dort aber zwei deutsche Soldaten und stehlen ihm sein Pferd. Olin weiß, dass er von nun an nicht mehr nach Hause zurückkehren kann, weil ihn der Vater nach dem Verlust des für das Überleben der Familie unentbehrlichen Pferdes totschlagen würde, und streift in der Gegend herum. Zunächst möchte er sich auf die gleiche Weise ein neues Pferd beschaffen und es den sowjetischen Soldaten stehlen, stattdessen freundet er sich mit ihnen an.

Die Atmosphäre der letzten Kriegstage wird sehr authentisch, kommentarlos und natürlich geschildert. Von der Ferne beobachtet Olin die Bewohner des Dorfes, wie sie den Gutshof eines reichen Bauern plündern. Als das Dorf befreit wird, sieht er, wie ein Mann aus dem Dorf, der andere Dorfbewohner denunziert hat, aus Rache von ihnen zum Selbstmord gezwungen wird. Schließlich nimmt Olin all seinen Mut zusammen und kehrt nach Hause zurück. Im Hof seiner Eltern stehen drei neue Pferde, und die Eltern laden gerade vom Fuhrwerk fremde Möbel aus.

Der Autor nennt das letzte Kapitel seines Buches „Was ich nie erfahren habe “. Es ist ein Nachwort zum Buch, in dem das Schicksal des Pferdes ein wenig rührend und sentimental geschildert wird. Deutsche Soldaten, die das Pferd von Olin gestohlen hatten, brachten das Tier nach Deutschland, wo es verlassen auf einem Marktplatz steht, die Menschen schauen es an und ein kleines Mädchen streichelt es.

Die historischen Ereignisse der letzten Wochen und Tage des Zweiten Weltkriegs werden in diesem Werk aus der Perspektive eines Jugendlichen geschildert. Nirgends wird jedoch direkt Anklage gegen den Krieg erhoben. Durch die Einbeziehung des Schicksals des Pferdes gelingt es Jan Procházka, eine neue, intime Erzählebene zu schaffen, die viel über das Verhältnis des Jungen zu dem Pferd aussagt. Frederik Hetmann (d. i. der Kinderbuchautor Hans-Christian Kirsch), der das Nachwort zu diesem Roman geschrieben hat, vergleicht gewisse Momente in der Handlung und die Charaktereigenschaften der Protagonisten dieses Werkes mit dem Roman Fänger im Roggen (englisch The Catcher in the Rye) von J. D. Salinger.

\section{Alena Santarová (1923-1967)}

Alena Santarová gehört zu den besten tschechischen Autoren der Mädchenliteratur der 60er Jahre (vgl. POLÁČEK: 2003). Bereits als Kind kam sie mit der Kinderliteratur in Berührung. Als Tochter des Klassikers der tschechischen Literatur, Vladislav Vančura, war sie nämlich dabei, als das fast allen tschechischen Kindern gut bekannte Buch Kubula a Kuba Kubikula (dt. 1971) entstand.

Im Jahre 1942 legte sie die Matura ab, wurde Schauspielerin und nach einem Studienaufenthalt in Paris arbeitete sie in der Filmbranche. Ab 1949 war sie als Redakteurin im Rundfunk, in Zeitungen und Zeitschriften beschäftigt, zuletzt im 
Verlag Čs. spisovatel und Státní nakladatelství dètské knihy, dem staatlichen Verlag für Kinderliteratur. Ihr erster Ehemann war der tschechische Regisseur Bořivoj Zeman.

Literarisch debütierte sie unter ihrem Mädchennamen Vančurová: Gemeinsam mit Ludvík Aškenazy und Stephan Hermlin veröffentlichte sie unter dem Titel Modrý zápisnik (1951) verschiedene Reportagen.

Im Jahre 1960 erschien das Buch Známá neznámá, ein belletristisch gestalteter Reiseführer durch Prag, wo Santarová die Prager Geschichte und Sehenswürdigkeiten vorstellt.

$\mathrm{Zu}$ ihrem Werk gehören weiter zwei Detektivgeschichten: Die eine, Od úterka do soboty (1963) war für Kinder bestimmt, die andere, Program na neděli (1966) für Erwachsene. In den bizarren Geschichten der Sammlung Slon v domácnosti (1965) versucht sie sich an der Gattung der Nonsens-Geschichte und knüpft damit an das Werk von Vladislav Vančura (Kubula a Kuba Kubikula) und Jindřich Plachtas (Pućálkovic Amina, beide zuletzt genannten Bücher erschienen 1931) an.

1980 erschien Káta, Katrin, Katynka in der ehemaligen DDR unter dem Titel Katja. Es ist eine autobiographisch gefärbte Feriengeschichte, die darüber hinaus Züge der Memoirenprosa aufweist. Die 15-jährige Protagonistin Káta verbringt die Ferien mit ihren Geschwistern, Cousins und Cousinen bei ihren Großeltern in Südböhmen. Sie bewertet alle bisherigen Beziehungen zu den Freundinnen und Geschwistern und entscheidet sich letztendlich, die gut gemeinten Ratschläge ihrer Großmutter hinsichtlich ihrer weiteren Bildung und des künftigen Berufs zu befolgen.

\section{Zusammenfassung:}

Der folgende Artikel befasst sich mit der Rezeption der tschechischen Kinder- und Jugendliteratur im Zeitraum zwischen 1945 und 1989 im deutschsprachigen Raum. In den fünfziger und sechziger Jahren wurden vor allem Werke der bedeutenden tschechischen Schriftsteller neu aufgelegt. Von den verschiedenen Genres war besonders das Märchen bzw. die Sage gefragt. In den siebziger und achtziger Jahren spielte (vor allem in der BRD) die phantastische Geschichte eine herausragende Rolle, wobei die Lektüre zunehmend durch den Film bzw. den Fernsehfilm ersetzt wurde. Die Kinderliteratur wurde generell gegenüber der Jugendliteratur bevorzugt. Am wenigsten wurde die Gattung der realistischen Mädchengeschichte rezipiert, weil sich in den entsprechenden Büchern deutlich die damals tonangebende sozialistische Ideologie - als die angeblich einzige richtige Lebensweise widerspiegelte. Einige Mädchenbücher wurden jedoch in der DDR übersetzt.

Schlüsselwörter:

Kinderliteratur - Jugendliteratur - Rezeptionsgeschichte - Übersetzungen - Kinderfilme Märchenfilme - Mädchengeschichten 


\section{Literatur:}

PROCHÁZKA, Jan. Aṫžije republika! Prag: SNDK 1966.

SANTAROVÁ, Alena. Káta, Katrin, Katynka. Prag: SNDK 1959.

SEIFERT/WEINKAUFF (2006): Seifert, Martina/Weinkauff, Gina. Tschechische Kinderund Jugendliteratur in deutscher Übersetzung, in: Seifert, Martina/Weinkauff, Gina Hg.): Kulturtransfer. Studien zur Repräsentanz einzelner Herkunftsliteraturen. München: Iudicium, 2006, 844-896.

HUTAŘOVÁ/HANZOVÁ (2003): Hutařová, Ivana/Hanzová, Marie. Současni češtíspisovatelé. Prag: Institut für Informationen in der Bildung, 2003.

CHALOUPKA (1985): Chaloupka, Otakar. Čeští spisovatelé literatury pro dèti a mládež. Prag: Albatros, 1985.

MORANSEE (1982): Moransee, Jess. R. (Hg.). Preisgekrönte Kinderbücher. München: K. G. Saur, 1982.

POLÁČEK (2003): Poláček, Jiří. Vzpominka na Alenu Santarovou. Ladění, Brno: ÚLM PdF 8, Nr. 2, 41-42, 2003. 